\title{
DIFICULTADES DE LENGUAJE EN NIÑOS DE 1 A 18 MESES DE EDAD
}

\section{LANGUAGE PROBLEMS IN CHILDREN FROMI TO 18 MONTHS}

Recibido septiembre 2015 Aceptado diciembre 2015 Publicado noviembre 2016

Correspondencia:

Dora E. Granados Ramos Manantial de San Cristóbal S/N $2^{\circ}$ piso Xalapa 2000. CP 91097 Xalapa, Ver. México Tel. 012288421700 Ext. 19518 dgranados@uv.mx

\section{Autores:}

Dora E. Granados Ramos

Universidad Veracruzana, Facultad de Psicología-Xalapa, Laboratorio de Psicobiología

Ana N. Figueroa Fernández

Universidad Veracruzana, Facultad de Psicología-Xalapa, Laboratorio de Psicobiología

Ma. Magdalena Álvarez Ramírez

Doctorado en Neuroetología, Universidad Veracruzana

Palabras clave: Dificultades de lenguaje, Evaluaciones de desarrollo, Niños

Key words: Language problems, Developmental Assessments, Children 


\section{RESUMEN}

Los niños aprenden el lenguaje desde etapas tempranas de la vida en la interacción con sus cuidadores principales y su medio ambiente. Existen factores de riesgo ambientales y biológicos que pueden dificultar el aprendizaje del lenguaje. Se realizó un estudio descriptivo, observacional, transversal con 18 niños (9 niñas y 9 niños) de 1 a 18 meses de edad de la ciudad de Xalapa, Veracruz Se evaluó el desarrollo general y del lenguaje con dos instrumentos: Evaluación de Desarrollo Infantil (EDI) y Escala de Desarrollo Infantil Bayley II (BSID-II). El objetivo fue identificar las dificultades del lenguaje de niños de 1 a 18 meses de edad. Se encontraron asociaciones significativas de los puntajes de lenguaje de la BSID-II con el Índice de Desarrollo Mental (IDM). En los niños de 1 a 24 meses de edad deben realizarse evaluaciones periódicas de lenguaje para prevenir dificultades posteriores en los aprendizajes básicos.

Palabras clave: Dificuttades de lenguaje, Evaluaciones de desarrollo, Niños

\section{ABSTRACT}

Children learn language from the early life stages when interacting with their primary caregivers and their environment. There are environmental and biological factors of risk that may compromise language learning. A descriptive, observational, cross-sectional study with 18 children (9 girls and 9 boys) from 1 to 18 months old in the city of Xalapa, Veracruz was performed. The general development and language was assessed with two instruments: Evaluation of Child Development (EDI) and Bayley Scales of Infant Development (BSID-ll). The aim was to identify the language difficulties of children from 1 to 18 months old. Significant associations of the language scores with the Mental Development Index (MDI) from BSID-II were found. In children aged 1 to 24 months old, periodic language assessments should be performed in order to prevent further difficulties in basic learning.

Keywords: Language problems, Developmental Assessments, Children 


\section{INTRODUCCIÓN}

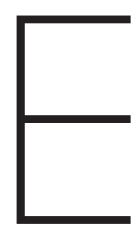

I lenguaje es un proceso característico del humano que se aprende en las etapas iniciales en interacción con la madre o los cuidadores principales y posteriormente con las personas que conforman una comunidad. Es resultado de factores biológicos y sociales; se conforma de aspectos fonológicos, semánticos, sintácticos y pragmáticos que se interrelacionan entre sí. En su desarrollo, se requiere del procesamiento sensorial para discriminar y analizar los sonidos que lo conforman, de la comprensión de las palabras y del uso adecuado de las reglas gramaticales.

Se consideran cuatro elementos prelingüísticos que favorecen el desarrollo del lenguaje: el contacto visual, los gestos, las expresiones corporales y las vocalizaciones. ${ }^{2}$ En las etapas tempranas se debe observar si dichos elementos están presentes en las interacciones cotidianas y recomendar a los cuidadores principales favorecer dichas conductas, debido a que, incluso en casos con audición normal se pueden observar dificultades en la adquisición del lenguaje. ${ }^{3}$

El lenguaje abarca cinco componentes estructurales: fonológico, semántico, sintáctico, morfológico y pragmático. El componente fonológico se relaciona con los sonidos y los patrones de entonación; el semántico con el significado de las palabras; el sintáctico con las reglas gramaticales en cuanto a la combinación de las palabras para formar frases u oraciones; el morfológico con las reglas que regulan el uso de morfemas o unidades más pequeñas con significado y el pragmático con la forma en la que los individuos utilizan su lenguaje de acuerdo al contexto en el que se encuentran y grupo social al que pertenecen., ${ }^{4,5}$

Antes de los tres años de edad se pueden observar algunas dificultades en la fluidez del lenguaje y en la articulación de fonemas como la /r/. En este periodo, la ausencia del lenguaje representa una alteración del desarrollo neurológico. El repertorio fonológico se desarrolla por completo, alrededor de los cuatro años de edad. ${ }^{\circ}$ A los cinco años, en la etapa preescolar, los niños que no presenten dificultades de lenguaje, dominarán los diferentes aspectos que lo componen, consolidarán los conocimientos previos e integrarán conocimientos nuevos para utilizar el lenguaje en tareas cada vez más complejas. ${ }^{5}$

Entre los indicadores tempranos de alguna dificultad del lenguaje se describen a los 10 meses, ausencia - pobreza del balbuceo; a los 18 meses, emisión de menos de 10 palabras diferentes; a los 24 meses, ausencia de enunciados con dos palabras: a los 30 meses, ausencia de enunciados de dos palabras; a los 36 meses, ausencia de enunciados de tres palabras con la estructura de sujeto -verbo -predicado?

Las alteraciones del lenguaje se definen en el Manual Estadístico de Enfermedades Mentales DSM-5, como trastornos de la comunicación con dificultades, fonológicas, de fluidez, de la comunicación social e incapacidad para usar el lenguaje verbal o no verbal para integrarse a un grupo social. ${ }^{8}$

En la Secretaría de Educación Pública (SEP) ? del total de niños de educación básica que se atienden en educación especial, el 9\% corresponde a niños con dificultades de comunicación. De acuerdo al Instituto Nacional de Estadístioa Geografíia e Informática $\left(\right.$ INEGI) ${ }^{10}$ en el censo de 2010, del total de niños menores de 5 años de edad, con limitaciones, el 32.3\%, presentan dificultades para hablar o comunicarse. Dichas cifras muestran la necesidad de evaluar el lenguaje en los primeros años de vida, para detectar y atender las dificultades que pueden asociarse a problemas motores y de atención, además de interferir en los aprendizajes escolares, específicamente en la adquisición de la lectoescritura. ${ }^{3,17}$

El objetivo de la investigación consistió en identificar las dificultades del lenguaje de niños de 1 a 18 meses de edad. 


\section{MATERIAL Y MÉTODOS}

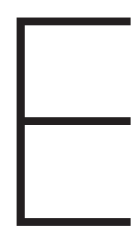

studio descriptivo, observacional, transversal con 18 niños (9 niñas y 9 niños) de 1 a 18 meses de edad de la ciudad de Xalapa. veracruz.

Se documentó el desarrollo general y del lenguaje con dos instrumentos: Escala de Desarrollo Infantil Bayley \| (BSID-II) ${ }^{12}$ y Evaluación de Desarrollo Infantil (EDI) ${ }^{13}$

Los padres de los niños firmaron una carta de consentimiento informado donde se les explicó el objetivo de la evaluación y se realizó una historia clínica. Los niños se evaluaron en presencia de sus madres o cuidadores principales, en un lugar ventilado, libre de distracciones.

La escala Bayley II (BSID-II) evalúa el desarrollo de los niños de 1 a 42 meses de edad y consta de tres escalas: mental, motora y de comportamiento; la escala mental consta de 178 reactivos, la psicomotora de 111 y la de comportamiento de 30. Los índices de confiabilidad de la BSID-II son de 87 para la escala mental y 78 para la escala psicomotora. La sensibilidad es de 0.75-0.90 y la especificidad de 0.76-0.91 respectivamente. ${ }^{14}$

En la BSID-II se clasifican los puntajes de desarrollo como: > a 115, Desempeño acelerado; de 85 a 114, Desempeño normal; de 70 a 84 Desempeño ligeramente retrasado y < 69 Desempeño significalivamente retrasado.

Para analizar los datos del lenguaje de la BSID-II, se establecieron los criterios de respuestas $<$ a 1 mes como desarrollo normal, las < 2 meses como rezago en el desarrollo y las > a 3 meses, como retraso en el desarrollo.

La prueba de Evaluación de Desarrollo Infantil (EDI) es una prueba de tamizaje de 1 a 60 meses de edad. Proporciona señales de alerta y alarma; evalúa las áreas de desarrollo motor, lenguaje, social, adaptativo y cognoscitivo. ${ }^{13}$
Para la calificación de las conductas observadas o reportadas por los padres o cuidadores principales, se califica si el niño tiene desarrollo normal, con rezago o con retraso, identificándolos con un sistema de semáforo como: a) Verde, desarrollo normal. Respuestas esperadas para la edad, no tiene señales de alarma ni alteración neurológica; b) Amarillo, rezago en el desarrollo. No realiza las actividades correspondientes a la edad, pero acredita las del grupo inmediato anterior del tamizaje y c) Rojo, probable retraso en el desarrollo. No acredita las conductas de su edad, ni de la edad anterior, tiene alteraciones neurológicas o presenta señales de alarma.

La sensibilidad de la prueba es de 81 y la especificidad de $27^{13}$

Se realizó un análisis descriptivo de las variables de estudio y para determinar la relación entre las variables se hizo un análisis de asociación para variables ordinales con los programas MINITAB 16 y EPI DAT 4.7.

Se consideraron los principios de la Declaración de Helsinki. ${ }^{75}$ Ninguno de los procedimientos puso en riesgo a los niños, prevaleció el respeto a la dignidad, la protección de los derechos y bienestar de los participantes.

\section{RESULTADOS}



n la escala mental del BSID-II, el 72\% tuvo desempeño normal y el 28\% ligeramente retrasado; en la escala psicomotora 44\% mostró desarrollo normal, 39\% ligeramente retrasado y $17 \%$ significativamente retrasado.

En la calificación de lenguaje la BSID-II identificó el $33 \%$ como normales, $11 \%$ con rezago y $56 \%$ con retraso, mientras que la EDI ubicó al 22\% como normales, 45\% con rezago y 33\% con retraso (Figura 1).

\section{2} MMN Why Enf Neurol Vol. 15. No. 1 enero - abril 2016 

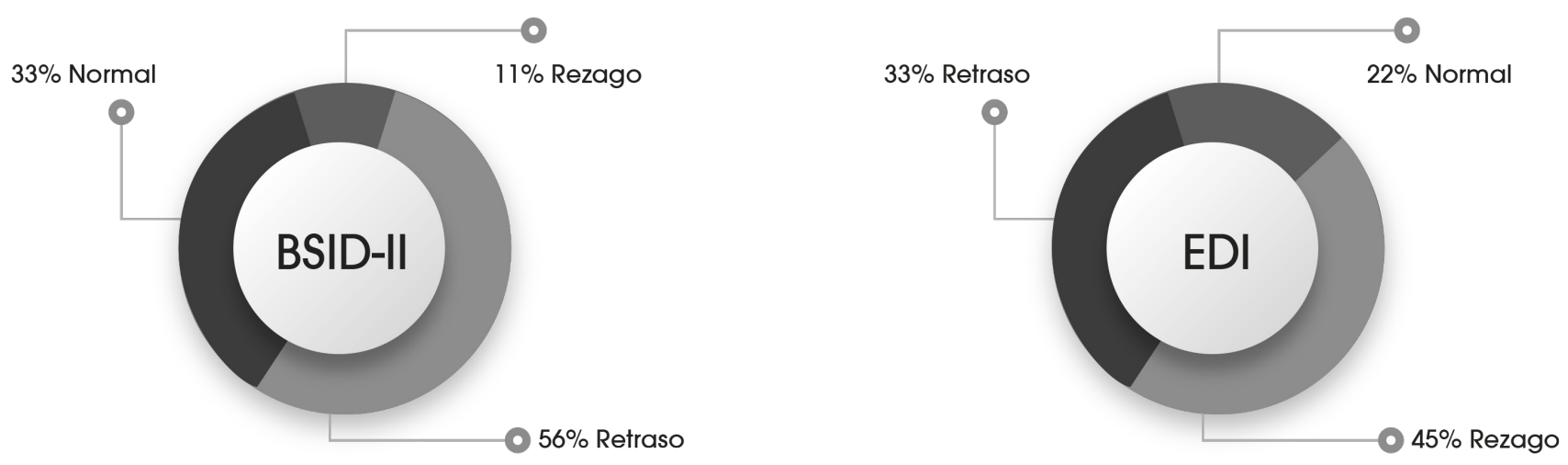

Figura 1. Porcentajes de niños detectados con desarrollo normal, rezago o retraso en el área de lenguaje con los instrumentos BSID-II y EDI.

En la comparación de resultados del lenguaje por sexo, en la BSID-ll el mayor número de casos correspondió a 6 niños que se ubicaron con retraso y en EDI a 4 para cada sexo, que se ubicaron con rezago (Tabla 1).

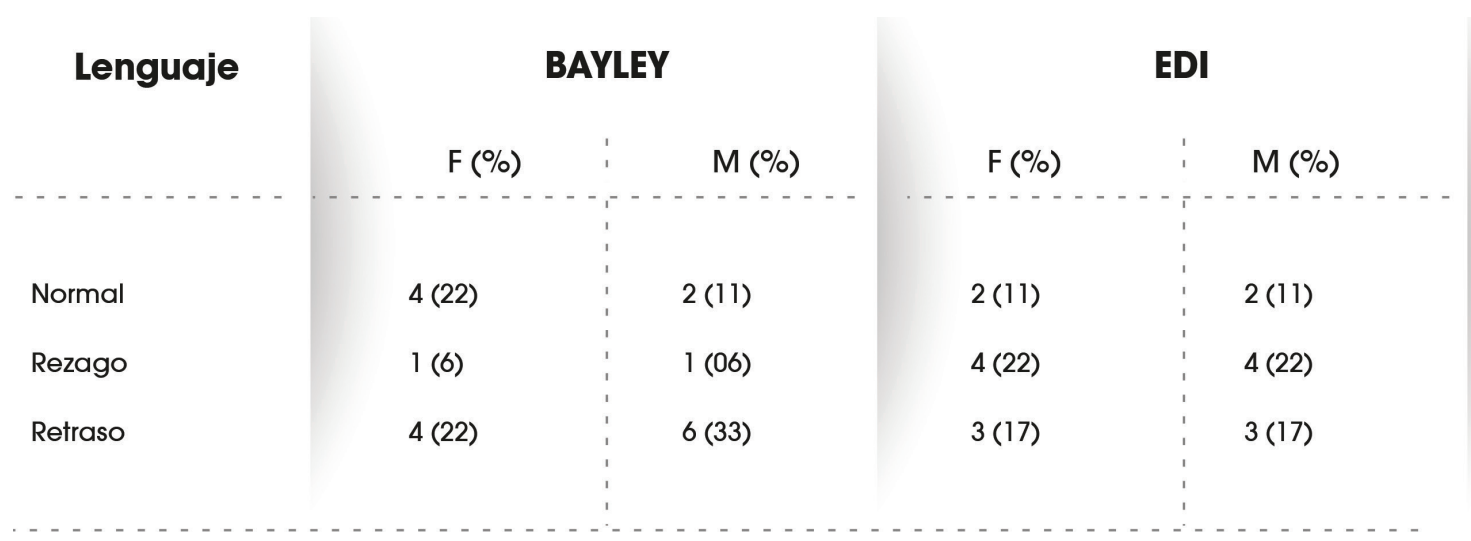

Fuente. Evaluación del Laboratorio de Psicología

El IDP comparado con los resultados de lenguaje no mostró correlación positiva, sin embargo la calificación obtenida en lenguaje de la BSID-II se relacionó de manera significativa con el resultado del IDM al realizar el análisis de asociación para variables ordinales (D Sommers .4675, p.00857). 


\section{DISCUSIÓN}

巨 n la actualidad, en los programas de salud se están implementando evaluaciones de desarrollo y programas de atención en etapas tempranas, debido a que mayor número de niños sobreviven gracias a los adelantos tecnológicos, sin embargo su morbi mortalidad dependerá de diversos factores postnatales cuya consecuencia será observable en los primeros dos años de vida o posteriormente en los aprendizajes básicos. ${ }^{16}$

Con esta investigación logramos el objetivo planteado de identificar las dificultades del lenguaje de niños de 1 a 18 meses de edad, mediante dos instrumentos de evaluación. Es necesario implementar estrategias terapéuticas oportunas a fin de disminuir la estructuración de secuelas del lenguaje, ya que se han reportado en la etapa escolar, la presencia de dificultades de lenguaje oral que pueden obstaculizar el aprendizaje del lenguaje escrito si no se atienden tempranamente. ${ }^{17}$

Los dos instrumentos utilizados no coincidieron en la calificación asignada al lenguaje, debido a que el BSID-|| calificó $17 \%$ más de niños normales y 23\% más con retraso que en la EDI. Considerando que la BSID-II es una escala de desarrollo que califica al niño, a partir de las respuestas ante tareas específicas a las que se enfrenta y EDI es una prueba de tamizaje que evalúa al niño, a partir de lo observado o reportado por los padres, debemos de considerar los resultados de BSID-l para derivar a una evaluación de lenguaje específica y apoyo terapéutico, en este caso al 56\% de los niños. De otra manera el 23\% de los niños se quedarían sin apoyo y la repercusión la observaríamos en las etapas escolares.

En poblaciones mexicanas se ha descrito que en las evaluaciones de lenguaje, un componente lingüístico: fonológico, léxico, morfosintáctico, semántico o pragmático, no es suficiente para identificar o explicar las dificultades de lenguaje que presenta un niño, es necesario analizar qué componentes están interactuando y complementarlo con las observaciones conductuales de un experto. ${ }^{18}$

Con las evaluaciones BSID-II y EDI se evaluó el desarrollo general de los niños y se identificaron los casos que presentaban rezago o retraso en lenguaje. aje donde se identifiquen los componentes lingüísticos que están comprometidos. La asociación significativa entre el Índice de Desarrollo Mental y el área de lenguaje en la escala BSID-II nos permitió confirmar cómo el profesionista a partir de los índices generales de desarrollo puede sugerir evaluaciones específicas de lenguaje a aquellos niños que tuvieron puntajes bajos en esa área y de allí derivarlos a atenciones oportunas que favorezcan la estimulación o la corrección de alteraciones en el lenguaje. No obstante deberá identificar si el área cognitiva, de lenguaje o de motricidad fina son las que están determinando los índices mentales bajos, para estructurar las estrategias adecuadas de intervención para cada uno de los casos.

Es necesario aumentar la muestra y comparar las respuestas por edad en los reactivos que evalúan lenguaje de los dos instrumentos, para identificar por qué no coinciden los casos calificados como normales, con rezago o retraso, de manera que se implementen estrategias de intervención oportunas que aminoren o prevengan la estructuración de secuelas de aprendizaje en las etapas escolares y por lo tanto se disminuyan los costos de atención.

\section{CONCLUSIONES}

I os niños que en los dos primeros años de vida se identifiquen con retrasos en el lenguaje deberán evaluarse con pruebas específicas para identificar los componentes lingüísticos L comprometidos así como recibir programas de intervención adecuados a cada caso para prevenir dificultades posteriores en los aprendizajes básicos. 


\section{REFERENCIAS BIBLIOGRÁFICAS}

1. Johnson, M.H. Developmental cognitive neuroscience. Third edition. UK: Wiley Blackwell. 2011.

2. Macarena, P. Adquisición del lenguaje. El principio de la comunicación. Cauce. Revista de Ïlología y su Didáctica. 2003:(6):321-347.

3. Webster, R. \& Shevell, M. Neurobiology of Specillc Language Impairment., J Child Neurol, 2004. 19:471fi481.

4. Munson, B., Edwards, J., \& Beckman, M. E. Phonological Knowledge in Typical and Atypical Speech and Language Development: Nature, Assessment, and Treatment. Minnesota: University of Minnesota. 2005

5. Brandone A.C., Salkind S.J., Golinkoff R.M., \& Hirsh-Pasek K.B. Language Development. In G. George G \& K.M. Minke (Eds.), Children's needs III: Development, prevention, and intervention. Washington DC: National Association of School Psychologists. 2006. pp. 499-514.

6. Ardila, A., Rosselli, M. Neuropsicología Clínica. la ed. México: Manual Moderno; 2007.

7. Narbona J. El lenguaje del niño y sus trastornos. En: N. Fejerman y E. Fernández- Álvarez Neurología Pediátrica 3 edición. Madrid-Buenos Aires: Panamericana. 2006.p. 1-12.

8. American Psychiatric Association (APA). Diagnostic and statistical manual of mental disorders: DSM-5. Fifth edition. Washington, DC: American Psychiatric. (2013).

9. Secretaria de Educación Pública (SEP), Orientaciones generales para el funcionamiento de los servicios de educación especial. México 2006.
10. Instituto Nacional de Estadística, Geografía e Informática (INEGI). Clasillcación de tipo de discapacidad. México (2010).

11. Sánchez C. Indicadores tempranos de los trastornos del desarrollo del lenguaje. AEPAP. 2010;203-4.

12. Bayley N. Bayley Scales of Infant Development. Second Edition (BSID-II). San Antonio: Hartcourt Brace \& Company; 1993.

13. Rizzoli, A., Schnaas, L., Liendo, S., Buenrostro-Márquez G. et al Validación de un instrumento para la detección oportuna de problemas de desarrollo en menores de 5 años en México. Medigraphic, 2013. 70(3): 195-208.

14. Pérez-López J, et al. Las escalas Bayley BSID-I frente a BSID-II como instrumento de evaluación en Atención Temprana. Anales de psicología. 2012; 28 (2): 484-489

15. World Medical Association. Declaration of Helsinki. Ethical principles for medical research involving human subjects (2013)

16. Gerometta G., Aspres N., Schapira I., Granovsky, G., \& Vivas, S. Evaluación a largo plazo del desarrollo psicomotor e intelectual de niños de 4 a 5 años de edad nacidos pretérmino y con muy bajo peso. Hospital Materno Infantil (2014). 33(1):2-7.

17. López S. La prevención de dillcultades del lenguaje oral en el marco escolar: Evaluación e intervención temprana. Pensamiento Psicológico (2005). 1(5):73-83.

18. Jackson-Maldonado D. La identil̈cación del Trastorno Especiîlco de Lenguaje en Niño Hispano-hablantes por medio de Pruebas Formales e Informales. Revista Neuropsicología, Neuropsiquiatría y Neurociencias (2011). 11(1):33-50. 